\title{
Piezoelectric energy harvesting from traffic-induced pavement vibrations
}

\author{
Xuezheng Jiang, ${ }^{1,2, a)}$ Yancheng $\mathrm{Li}^{2}{ }^{2}$ Jianchun $\mathrm{Li}^{2}{ }^{2}$ Jiong Wang, ${ }^{3}$ and Jin Yao ${ }^{1}$ \\ ${ }^{1}$ School of Manufacturing Science and Engineering, Sichuan University, \\ No. 24 South Section 1, Yihuan Road, Chengdu 610065, China \\ ${ }^{2}$ Centre for Built Infrastructure Research, Faculty of Engineering and Information \\ Technology, University of Technology Sydney, P.O. Box 123, 15 Broadway, Sydney, \\ New South Wales 2007, Australia \\ ${ }^{3}$ School of Mechanical Engineering, Nanjing University of Science and Technology, \\ 200 Xiaolingwei Street, Xuanwu District, Nanjing 210094, China
}

(Received 21 January 2014; accepted 11 July 2014; published online 24 July 2014)

This paper focuses on the development and experimental testing of a potential clean energy source for powering the remote equipment used in transportation infrastructure. Traditional power sources (i.e., power cables and batteries) are excessively expensive or infeasible in this type of application. A compressionbased roadway energy harvester has been developed that can be embedded into pavement to scavenge electrical energy from traffic-induced vibrations. The proposed roadway harvester employs a group of piezoelectric harvesting units to convert traffic-induced vibrations into electrical energy, and each single harvesting unit contains three piezoelectric multilayer stacks. According to the linear theory of piezoelasticity, a two-degree-of-freedom electromechanical model of the piezoelectric harvesting unit was developed to characterize its performance in generating electrical energy under external excitations. Experimental testing in the laboratory was conducted to investigate the output power properties of the harvesting unit and shows good agreement with the theoretical analysis. Based on the testing results of the harvesting unit, the capability of the proposed roadway harvester has been theoretically evaluated and demonstrated that it has the ability to generate sufficient energy for driving common electrical equipment used in transportation infrastructure. (C) 2014 AIP Publishing LLC. [http://dx.doi.org/10.1063/1.4891169]

\section{INTRODUCTION}

In recent years, the remote electrical equipment has becoming ubiquitous in transportation infrastructure, such as the application of wireless sensors to improve the safety, management, and smooth operation of the transportation system. ${ }^{1,2}$ With the context of wireless sensors in the transportation system, one of the key challenges is the limit of sustainable and reliable power source, especially for remote sensing networks. With a vast number of sensors nodes/networks in the infrastructure, simply connecting them to the grid power source is uneconomical in the era of wireless technology. Moreover, using batteries as a source of power for dense sensor networks in the road may not be feasible due to the continuous replacement requirement. ${ }^{3}$ Besides, the disposal of large quantities of batteries may also create serious environmental hazards. Therefore, a sustainable and environmental-friendly energy source is required to fulfill the urgent requirement by the transportation industry. In this study, a potential clean and reliable energy source for the wireless sensor network is developed to scavenge energy from traffic-induced vibrations.

Extracting electrical energy from ambient vibrations is generally known as vibration energy harvesting, or vibration energy scavenging. Williams and Yates ${ }^{4}$ have stated that three basic

\footnotetext{
a) Author to whom correspondence should be addressed. Electronic mail: xuezheng.jiang@hotmail.com. Telephone: +8613980097279 .
} 
methods, i.e., electromagnetic induction, ${ }^{5}$ electrostatic generation, ${ }^{6}$ and piezoelectric effect, ${ }^{7}$ could be utilized to scavenge electrical energy from ambient vibrations. While each of the aforementioned methods can generate a useful amount of energy, piezoelectric vibration energy harvesters have received more attentions due to their ability to directly convert applied force into usable electrical charge, as well as its large power density, and ease of application. ${ }^{8}$ To date, piezoelectric vibration energy harvesters have been developed in various types of structures for different purposes. ${ }^{7,9}$ In recent years, a number of studies have begun to focus on harvesting energy from traffic-induced vibrations in transportation infrastructures. In the paper of Peigney and Siegert, ${ }^{10}$ the use of a cantilever piezoelectric harvester to scavenge energy from traffic-induced bridge vibrations was theoretically analyzed and experimentally investigated. The cantilever piezoelectric harvester was attached on a water pipe fixed on bridge girder, and the maximum energy generated was found to be in the order of $0.03 \mathrm{~mW}$, with a controlled voltage between 1.8 and $3.6 \mathrm{~V}$. Wischke et al. ${ }^{11}$ have investigated the feasibility of harvesting energy from traffic-induced vibrations in railway and road tunnels to power embedded structural health monitoring sensors. They concluded that the traffic-induced vibrations at any location in the road tunnel and at the wall in the railway tunnel are too small for useful vibration harvesting by using the cantilever-based piezoelectric generator. Xiong et al. ${ }^{12}$ installed the piezoelectric harvester inside the road and studied the feasibility of harvesting energy from trafficinduced deformation of pavements. They presented a general philosophy of designing and evaluating energy harvesting systems using piezoelectric materials from deforming pavements and concluded that the theoretical harvested energy is suitable for powering small-electronics used in transportation infrastructures. Zhao et al. ${ }^{13,14}$ have compared the performance of several piezoelectric transducers embedded inside the road for harvesting energy from asphalt pavement. They concluded that piezoelectric transducers fabricated in multilayer, cymbal, and bridge have high durability and can fit the load repetition in the pavement life. Using infinite BernoulliEuler beam model of the pavement, Xiang et al. ${ }^{15}$ have recently theoretical estimated the electrical energy generated by a piezoelectric harvester from traffic induced deformation of pavements and concluded that the velocity of the vehicle is a critical factor affecting the output power.

Grounded on the fore fundamental studies, this paper presents a novel compression-based roadway energy harvester to provide energy for the remote electrical equipment used in transportation infrastructures, such as wireless sensors and sensor networks. The novel roadway energy harvester will be installed in-between the road pavement and endures the tire forces of passing vehicles directly. According to the positive piezoelectric effect, the proposed roadway harvester employs a group of piezoelectric harvesting units (each one contains three piezoelectric multilayer stacks) to convert traffic-induced vibrations into electrical energy. More precisely, this paper is organized as follows. Section II briefly introduces the design of the proposed roadway harvesting system. Section III provides the theoretical analysis on the output voltage and power properties of the piezoelectric harvesting unit. Then, lab-scale tests are conducted to experimentally evaluate the theoretical analysis and investigate the energy harvesting capability of the piezoelectric harvesting unit in Sec. IV. Based on the testing results of the harvesting unit, Sec. V theoretically estimates the electrical energy harvested by the proposed roadway harvester on a highway with 2000 vehicle/h traffic volume and $100 \mathrm{~km} / \mathrm{h}$ velocity. Finally, conclusions from this study are drawn in Sec. VI.

\section{ROADWAY ENERGY HARVESTING SYSTEM DESIGN}

Fig. 1 schematically depicts the implementation of the proposed roadway energy harvester on the road. As shown in Fig. 1, several roadway energy harvesters are embedded on the surface of the road, and they will generate electrical energy when a car or a truck passes over. The energy management unit is used to convert the generated energy into a useful form that can be utilized directly by the nearby energy utilization system, such as wireless sensor networks that provide information about road conditions, traffic usage and patterns, and vehicle speeds. Optionally, energy storage unit, such as large capacitor or, preferably, a rechargeable 


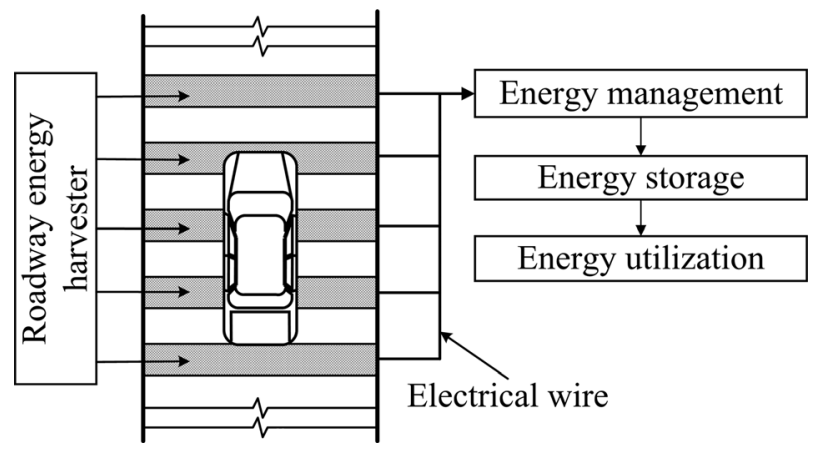

FIG. 1. Roadway energy harvesting system.

battery, is used for storing the harvested energy to be used when needed. Since the generated energy is present only when cars passes over the energy harvesting system, energy storage will be useful, so that the power supply is not interrupted when cars are absent or traffic is low.

The proposed roadway energy harvester is a compression-based system, which generates energy under compression force, and the generated power is a pulse power registered with each compression cycle. Fig. 2 shows the side cross-sectional view of the proposed roadway harvester embedded on the surface of the road, $F_{\text {left }}$ and $F_{\text {right }}$ are used to present the tire forces caused by one single axle of a car. As shown in Fig. 2, the proposed roadway harvester is a box shaped and chiefly comprised of three harvesting units, three force transmission flanges, a rigid compressing plate, and a force anchoring plate. Rigid compressing plate, combined with force transmission flange and force anchoring plate, ensures that the tire forces exert evenly on three harvesting units. Two elastic fixing members such as bolts are used to hold the structure together and applied a pre-compression force to the harvesting units. When cars pass over, the pressure and vibration caused by the moving vehicle create time variant forces on harvesting units which generate electrical power. It should be noted that the roadway harvester is pre-compressed so that electricity will still be generated during the rebounding of the compression.

In practice, three harvesting units are electrically connected to each other to scavenge electrical energy via electrical wire, and a bolt is used to connect the harvesting unit to the force anchoring plate through the axial hole of the harvesting unit. Each single harvesting unit contains three piezoelectric multilayer stacks (which can also be extended), as shown in Fig. 3. The piezoelectric multilayer stack is made up of several very thin piezoelectric wafers and electrode elements, as shown in Fig. 4. There are two ceramic layers attached at the bottom and top of the stack to protect from damage under large compression force caused by the axle weight of vehicles. The piezoelectric wafers are polarized along this thickness direction, and all are assembled mechanically in series with alternative polarization direction as depicted by the arrows but connected electrically in parallel.

It needs to note that vehicles on the road are free to change lanes at will, and are also able to vary their position within a lane to some degree. Therefore, there is some probability that

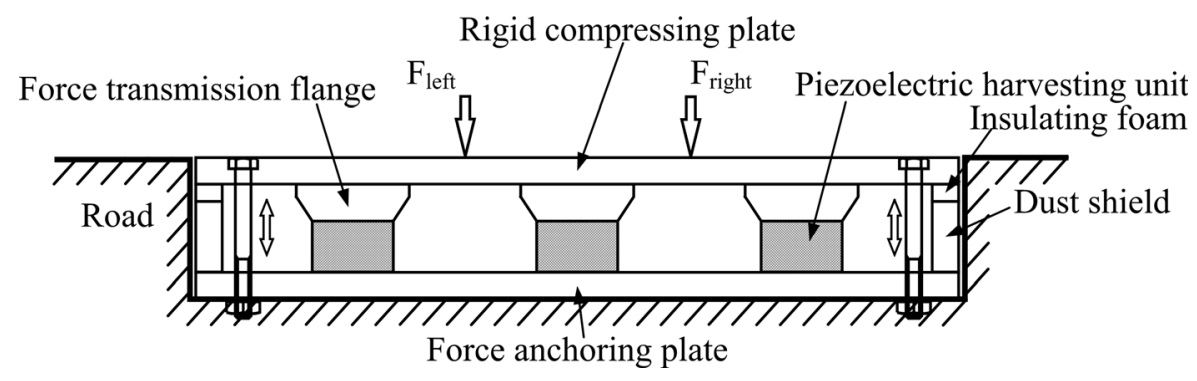

FIG. 2. Schematic of the proposed roadway harvester. 


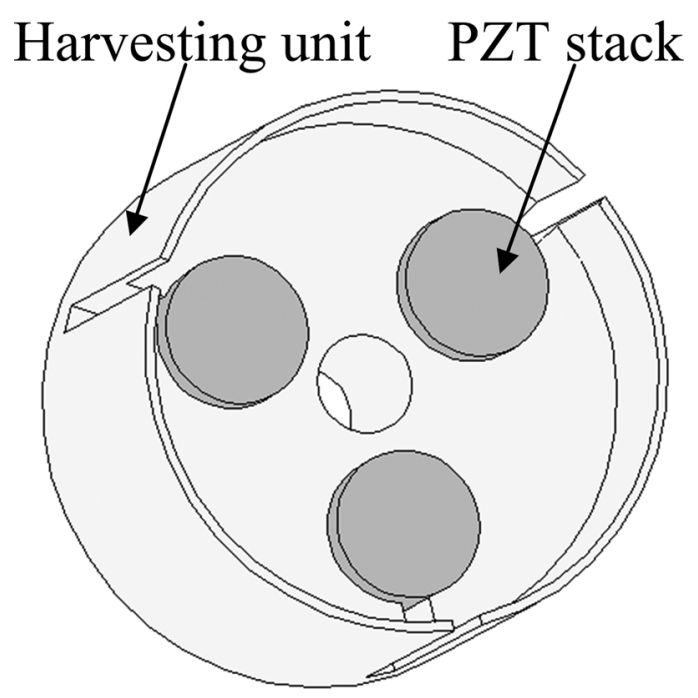

FIG. 3. Schematic of the harvesting unit.

when energy harvesters are implanted in the road, passing vehicles will not apply compression to the devices due to lane changes or asymmetrical positions within the lane. To eliminate the possible idle passing, the roadway harvester proposed in this paper uses a wide rigid plate, which covers the whole width of road, to receive tire forces of passing vehicles. Therefore, all passing vehicles will impart energy to the energy harvesting system in this design. Also, as stated by Zhao et al. ${ }^{13}$ that the piezoelectric transducers had better locate in the pavement as close as possible to the surface to get more energy, therefore, the proposed harvester employs a novel structure and embeds on the surface of the road to directly respond to the tire forces of each passing vehicle.

\section{THEORETICAL ANALYSIS FOR THE PIEZOELECTRIC HARVESTING UNIT}

The proposed roadway harvester employs piezoelectric harvesting units to scavenging energy from traffic-induced vibrations. For evaluating the energy harvesting capability of the roadway harvester, it is necessary to investigate the electrical characteristics and energy harvesting properties of the piezoelectric harvesting unit under external excitation. According to the IEEE Standard on Piezoelectricity, ${ }^{16,17}$ under the time variant exciting pressure, given by the strain $S$, stress $T$, electric field $E$, and electric displacement $D$, the constitutive relations of piezoelectric materials are typically defined by

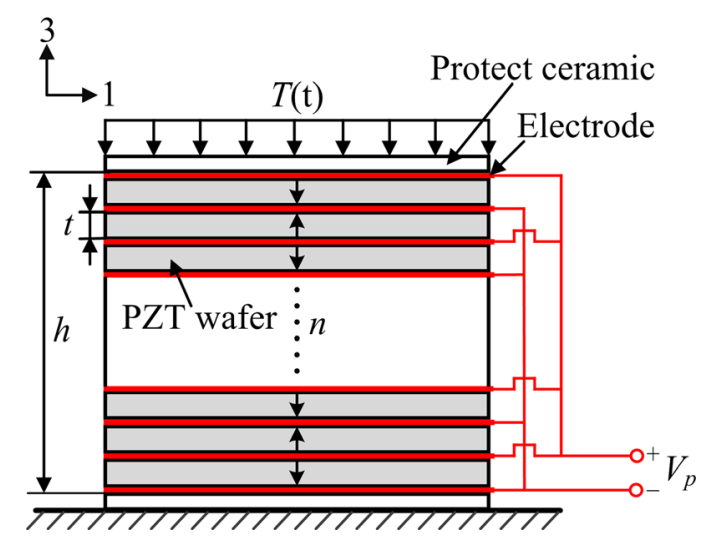

FIG. 4. Schematic of the piezoelectric stack. 


$$
\left[\begin{array}{c}
T_{3} \\
D_{3}
\end{array}\right]=\left[\begin{array}{cc}
c_{3}^{E} & -e_{33} \\
e_{33} & \varepsilon_{33}^{S}
\end{array}\right]\left[\begin{array}{l}
S_{3} \\
E_{3}
\end{array}\right]
$$

where the $e_{33}$ is the piezoelectric coefficient; $c^{E}{ }_{3}$ is the elastic stiffness constant under a constant electric field; and $\varepsilon_{33}^{S}$ is the dielectric constant under constant strain.

For the piezoelectric stack shown in Fig. 4, the parameters in Eq. (1) can be calculated as

$$
S_{3}=x_{2} / h, \quad E_{3}=V_{p} / t, \quad T_{3}=F_{p} / A, \quad D_{3}=Q / n A,
$$

where $h$ is the height of the stack; $A$ is the cross-section area of the stack; $t$ is the thickness of a single piezoelectric wafer; $F_{p}$ is the time variant force applied on the stack; $x_{2}$ is the deformation of the stack under the exciting force; $Q$ is the electric charge generated by the stack; $V_{p}$ is the output voltage of the stack; and $n$ is the number of piezoelectric wafers used to produce the stack.

In order to simplify the analysis, let the height of the stack be equal to the entire thickness of all the piezoelectric wafers (i.e., $h=n t$ ). Then, substitution of Eq. (2) into Eq. (1) yields the constitutive equations of the piezoelectric stack as

$$
\left[\begin{array}{c}
F_{p} \\
Q
\end{array}\right]=\left[\begin{array}{cc}
k_{2} & -N \\
N & C_{2}
\end{array}\right]\left[\begin{array}{l}
x_{2} \\
V_{p}
\end{array}\right]
$$

In Eq. (3), $k_{2}$ is the elastic coefficient of the stack $\left(k_{2}=c_{3}^{E} A / h\right), C_{2}$ represents the equivalent capacitance of the stack $\left(C_{2}=n \varepsilon_{33}^{S} A / t\right)$, and $N$ is defined as the electromechanical conversion coefficient of the stack $\left(N=e_{33} A / t\right)$. The electromechanical conversion coefficient $N$ represents the force-voltage and charge-deformation transferring relation of the stack. From Eq. (3), a converted force, which actually converts mechanical energy into electrical energy, can be written as

$$
F_{e}=N V_{p}
$$

Fig. 5 develops a two-degree-of-freedom (2-DOF) electromechanical model of the piezoelectric harvesting unit under the exciting force. As shown in Fig. 5, the 2-DOF electromechanical model considers: (1) the time variant exciting force $F(t)$; (2) mechanical responses of the harvesting unit (i.e., $x_{1}$ and $x_{2}$ represent the deformations of the ceramic layers and piezoelectric stack); (3) mechanical properties of the harvesting unit (i.e., $m_{1}, c_{1}$, and $k_{1}$ represent mechanical properties the ceramic layers; $m_{2}$ and $k_{2}$ represent mechanical properties the piezoelectric stack; $c_{3}$ and $k_{3}$ represent mechanical properties of mechanical structure of the harvesting unit); (4) electrical properties of the harvesting unit (i.e., $R_{2}$ and $C_{2}$ represent the leakage resistance and capacitance of the piezoelectric stack); (5) the energy management circuit (i.e., an AC-DC rectifier with a smoothing capacitor $C_{3}$ ); (6) the external electrical loading (i.e., $R_{3}$ ); and (7) the output voltage on the electrical loading (i.e., V).

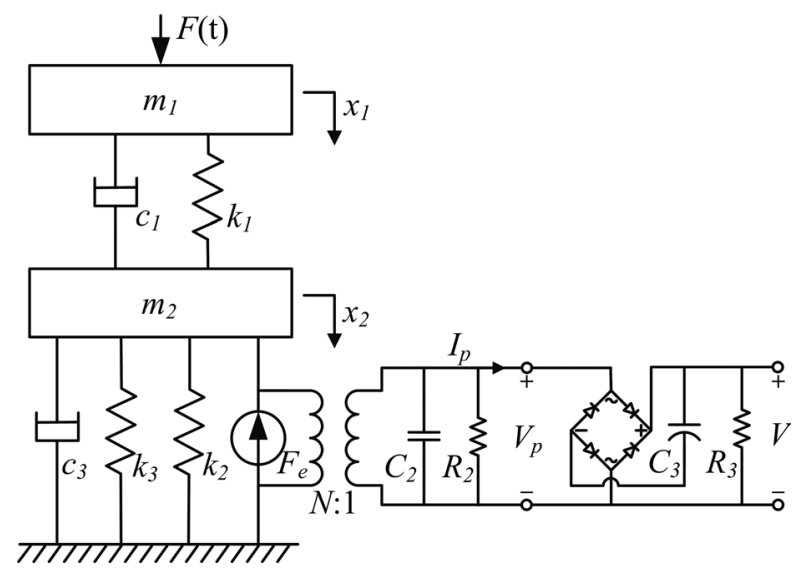

FIG. 5. 2-DOF electromechanical model of the piezoelectric harvesting unit. 
In the developed 2-DOF electromechanical model, $F_{e}$ is used to link the mechanical parts and electrical parts of the model and convert mechanical energy into electrical energy. The governing equations of the electromechanical model can be written as

$$
\left\{\begin{array}{l}
m_{1} \ddot{y}+c_{1} \dot{y}+k_{1} y=F(t)-m_{1} \ddot{x}_{2}, \\
m_{2} \ddot{x}_{2}+c_{3} \dot{x}_{2}+k_{3} x_{2}+k_{2} x_{2}+F_{e}=F(t)-m_{1} \ddot{x}_{2}-m_{1} \ddot{y}, \\
-N \dot{x}_{2}+C_{2} \dot{V}_{p}+V_{p} / R=0
\end{array}\right.
$$

where $y$ is defined as $y=x_{1}-x_{2}$; and $R$ is the equivalent resistance of two parallel resistances $R_{2}$ and $R_{3}$. In general, the $R_{2}$ is much higher than the loading resistance, so that $R \approx R_{3}$.

Transforming Eq. (5) into the frequency domain, one can obtain

$$
\left\{\begin{array}{l}
\left(-\omega^{2}+2 \zeta_{1} \omega_{1} \omega j+\omega_{1}^{2}\right) Y(\omega)-\omega^{2} X_{2}(\omega)=F(\omega) / m_{1}, \\
{\left[-(1+\mu) \omega^{2}+2 \zeta_{2} \omega_{2} \omega j+\omega_{2}^{2}\right] X_{2}(\omega)-\mu \omega^{2} Y(\omega)+\frac{N V_{p}(\omega)}{m_{2}}=\frac{F(\omega)}{m_{2}},} \\
-\omega N j X_{2}(\omega) / C_{2}+\left(\omega j+1 / R_{3} C_{2}\right) V_{p}(\omega)=0 .
\end{array}\right.
$$

Here, $\omega$ is the angular frequency of the vibration; $Y(\omega), X_{2}(\omega), V_{p}(\omega)$, and $F(\omega)$ are the frequency counterparts of $y, x_{2}, V_{p}$, and $F(t)$. Other parameters, i.e., $\omega_{1}, \omega_{2}, \zeta_{1}, \zeta_{2}$, and $\mu$, are defined as

$$
\omega_{1}=\sqrt{\frac{k_{1}}{m_{1}}}, \quad \omega_{2}=\sqrt{\frac{k_{2}+k_{3}}{m_{2}}}, \quad \zeta_{1}=\frac{c_{1}}{2 \sqrt{m_{1} k_{1}}}, \quad \zeta_{2}=\frac{c_{3}}{2 \sqrt{m_{2}\left(k_{2}+k_{3}\right)}}, \quad \mu=\frac{m_{1}}{m_{2}} .
$$

Solving Eq. (6), we obtain

$$
\begin{gathered}
X_{2}(\omega)=\frac{\frac{\left(\alpha^{2}+2 \zeta_{1} \psi \alpha j\right)}{\left(k_{2}+k_{3}\right)\left(\alpha^{2}-\psi^{2}+2 \zeta_{1} \psi \alpha j\right)} F(\omega)}{1-(1+\mu) \psi^{2}+2 \zeta_{2} \psi j-\frac{\mu \psi^{4}}{\alpha^{2}-\psi^{2}+2 \zeta_{1} \psi \alpha j}+\frac{\beta \psi N_{e}^{2} j}{1+\beta \psi j}}, \\
V_{p}(\omega)=\frac{\frac{\left(\alpha^{2}+2 \zeta_{1} \psi \alpha j\right)}{N\left(\alpha^{2}-\psi^{2}+2 \zeta_{1} \psi \alpha j\right)} F(\omega)}{\left[1-(1+\mu) \psi^{2}+2 \zeta_{2} \psi j-\frac{\mu \psi^{4}}{\alpha^{2}-\psi^{2}+2 \zeta_{1} \psi \alpha j}\right] \frac{1+\beta \psi j}{\beta \psi N_{e}^{2} j}+1},
\end{gathered}
$$

where $\alpha$ and $\psi$ are the normalized frequencies; $\beta$ is the normalized electrical resistance; and $N_{e}$ is the alternative electromechanical conversion coefficient. Those dimensionless parameters can be calculated as

$$
\alpha=\frac{\omega_{1}}{\omega_{2}}, \quad \psi=\frac{\omega}{\omega_{2}}, \quad \beta=\omega_{2} R_{3} C_{2}, \quad N_{e}^{2}=\frac{N^{2}}{C_{2}\left(k_{2}+k_{3}\right)} .
$$

In practice, the smoothing capacitor $C_{3}$, as shown in Fig. 5, is chosen to be large enough so that the time constant $R_{3} C_{3}$ is much larger than the oscillating period of the exciting vibration. As a result, the output rectified voltage $V$ can be considered as a constant DC voltage. Then, the current flowing into the rectified circuit can be calculated as

$$
I_{p}= \begin{cases}C_{3} \dot{V}+V / R_{3} & \text { if } V_{p}=V \\ -C_{3} \dot{V}-V / R_{3} & \text { if } V_{p}=-V \\ 0 & \text { if }\left|V_{p}\right|<V\end{cases}
$$


Based on Eq. (11), the third equation of Eq. (5) can be rewritten as Eq. (12) to investigate the output current and DC voltage on the electrical loading

$$
-N \dot{x}_{2}+C_{2} \dot{V}_{P}+I_{p}=0 .
$$

Therefore, it can be found that the output voltage $V_{p}$ of the piezoelectric stack varies proportionally with respect to the stack deformation $x_{2}$ if the rectifier bridge is blocked and the outgoing current is zero. Defining $T=2 \pi / \omega$ as the period of the vibration, and $t_{1}$ and $t_{2}$ as two time instants $\left(t_{2}-t_{1}=T / 2\right)$, thus, the strain $x_{2}$ goes from the minimum $-x_{2 m}$ to the maximum $x_{2 m}$ ( $x_{2 m}$ is the constant magnitude of the deformation). Assume that $\dot{V}_{p} \geq 0$ during the time period from $t_{1}$ to $t_{2}$; therefore, the integration of Eq. (12) from time $t_{1}$ to $t_{2}$ is

$$
-2 N x_{2 m}+2 C_{2} V+\frac{T}{2} \frac{V}{R_{3}}=0 .
$$

Then, based on Eq. (13), the output voltage $V$ can be expressed as a function of the deformation amplitude $x_{2 m}$,

$$
V=\frac{2 \omega N R_{3}}{2 \omega C_{2} R_{3}+\pi} x_{2 m}
$$

Assume that the external force excitation is independent of the piezoelectric harvester. Then, based on Eq. (8), the deformation amplitude $x_{2 m}$ can be calculated as

$$
x_{2 m}=\frac{F_{m} \alpha}{\left(k_{2}+k_{3}\right)\left(A_{3}^{2}+A_{4}^{2}\right)} \sqrt{A_{5}^{2}+A_{6}^{2}},
$$

where $F_{m}$ is the amplitude of the exciting force, and the parameters $A_{1}$ to $A_{6}$ can be calculated as

$$
\begin{gathered}
\left\{\begin{array}{l}
A_{1}=\alpha^{2}-\psi^{2}-(1+\mu) \alpha^{2} \psi^{2}+\psi^{4}-4 \zeta_{1} \zeta_{2} \psi^{2} \alpha \\
A_{2}=2 \psi\left[\alpha\left(1-\psi^{2}-\mu \psi^{2}\right) \zeta_{1}+\left(\alpha^{2}-\psi^{2}\right) \zeta_{2}\right]
\end{array}\right. \\
\left\{\begin{array}{l}
A_{3}=A_{1} \beta \psi+\left(\alpha^{2}-\psi^{2}\right) \beta N_{e}^{2} \psi+A_{2} \\
A_{4}=A_{2} \beta \psi+2 \zeta_{1} \alpha \beta N_{e}^{2} \psi^{2}-A_{1}
\end{array}\right. \\
\left\{\begin{array}{l}
A_{5}=\psi \beta\left(A_{3} \alpha+2 A_{4} \zeta_{1} \psi\right)+2 A_{3} \zeta_{1} \psi-A_{4} \alpha \\
A_{6}=\psi \beta\left(2 A_{3} \zeta_{1} \psi-A_{4} \alpha\right)-2 A_{4} \zeta_{1} \psi-A_{3} \alpha
\end{array}\right.
\end{gathered}
$$

Then, the output DC voltage under the external resistive load can be rewritten as

$$
V=\frac{2 \omega N R_{3}}{2 \omega C_{2} R_{3}+\pi} x_{2 m}=\frac{2 N_{e}^{2} \beta \psi \alpha \sqrt{A_{5}^{2}+A_{6}^{2}}}{N\left(A_{3}^{2}+A_{4}^{2}\right)(2 \psi \beta+\pi)} F_{m} .
$$

Based on Eq. (19), the electrical power under the external resistive load can be expressed as

$$
P=\frac{V^{2}}{R_{3}}=\frac{4\left(A_{5}^{2}+A_{6}^{2}\right) N_{e}^{4} \beta^{2} \psi^{2} \alpha^{2} F_{m}^{2}}{\left(A_{3}^{2}+A_{4}^{2}\right)^{2}(2 \psi \beta+\pi)^{2} N^{2} R_{3}} .
$$

Equation (20) indicates that the electrical power, generated by the piezoelectric harvesting unit, depends on the exciting vibration characteristics (the normalized frequency $\psi$ and amplitude $F(\omega)$ ), the mechanical coefficients of the harvesting unit (such as the natural frequency $\omega_{1}$ and 
$\omega_{2}$, the mechanical damping factor $\zeta_{1}$ and $\zeta_{2}$, and the stiffness of the harvester $k_{3}$ ), and the electrical properties of the harvesting unit (such as the normalized electrical load $\beta$, and the electromechanical conversion coefficient $N_{e}$ ). Based on Eqs. (10), (17), and (18), it can be found that the value of parameters $\mathrm{A} 3-\mathrm{A} 6$ and $\beta$ are all relate to the external electrical resistance $R_{3}$. Then, Eqs. (19) and (20) indicate that the value of the external electrical loading has a great impact on the output voltage $V$ and power $P$. It is of great need to find out the optimal external resistive load, on which the power generated by the harvester reaches its maximum value. Based on Eq. (20), the optimal resistive load and the maximum electrical power generated by the harvester can be calculated as

$$
\begin{aligned}
& R_{o p t}=\frac{\pi A}{2\left(A+\alpha^{2} N_{e}^{2}-\psi^{2} N_{e}^{2}\right) C_{2} \omega} \text { and } \\
& P_{\max }=\frac{\left[\left(3.5 A+\alpha^{2} N_{e}^{2}-\psi^{2} N_{e}^{2}\right)^{2}+2.5\left(\alpha^{2} N_{e}^{2}-\psi^{2} N_{e}^{2}\right)\right] \alpha^{4} N_{e}^{2} F_{m}^{2} \omega}{6 \pi A\left(k_{2}+k_{3}\right)\left(2 A+\alpha^{2} N_{e}^{2}-\psi^{2} N_{e}^{2}\right)^{2}\left(A+\alpha^{2} N_{e}^{2}-\psi^{2} N_{e}^{2}\right)},
\end{aligned}
$$

where $A=\alpha^{2}-\psi^{2}-(1+\mu) \alpha^{2} \psi^{2}+\psi^{4}$.

Zhao et al. ${ }^{13}$ have indicated that the frequency of the traffic-induced vibration is low $(0.1-10 \mathrm{~Hz})$. Since the natural frequency of the piezoelectric unit, $\omega_{2}$, is normally very high (equals to $1.78 \mathrm{e} 4 \mathrm{~Hz}$ in this design). Therefore, based on Eq. (10), it is reasonable to assume that the normalized frequency $\psi$ can be set to zero in traffic-induced vibration situation. Under this assumption, the optimal resistive load and the maximum generated power can be simplified as

$$
R_{\text {opt }}=\frac{\pi}{2\left(1+N_{e}^{2}\right) C_{2} \omega} \quad \text { and } \quad P_{\max }=\frac{\left[\left(3.5+N_{e}^{2}\right)^{2} \alpha^{2}+2.5 N_{e}^{2}\right] N_{e}^{2} F_{m}^{2} \omega}{6 \pi \alpha^{2}\left(k_{2}+k_{3}\right)\left(2+N_{e}^{2}\right)^{2}\left(1+N_{e}^{2}\right)}
$$

Equation (22) indicates that the proposed piezoelectric harvester is a linear system, in which output power increases with the input amplitude and frequency, and the maximum generated power is proportional to the exciting frequency and proportional to the square of the amplitude of excitation. Meanwhile, Eq. (22) shows that the optimal electrical loading is not a constant value and changes its value with the property of the piezoelectric material and the input frequency. For a weak electromechanical coupling coefficient $N_{e}$, the optimal resistance can be simplified as $\pi / 2 C_{2} \omega$.

\section{SINGLE HARVESTING UNIT OUTPUT POWER EXPERIMENTAL EVALUATION}

\section{A. Experimental setup}

Experimental testing is conducted to evaluate a single harvesting unit thus build knowledge for the performance of proposed roadway harvester. Fig. 6 shows the test specimens of piezoelectric stacks and harvesting unit. Each piezoelectric stack is made of 36 layers of piezoelectric wafers (PZT-8), 37 layers of brass electrodes, and 2 layers of protective ceramic. These 37 layers of brass electrodes and 36 layers of piezoelectric wafers are constructed together alternatively. All the piezoelectric layers are polarized along their thickness direction and are assembled mechanically in series but connected electrically in parallel, as shown in Fig. 4, in order to decrease the amplitude of output AC voltage and increase the output DC power. Three piezoelectric stacks, as shown in Fig. 6, are electrically connected in parallel to each other in the harvesting unit to generate electrical energy simultaneously. The properties of the piezoelectric stack are shown in Table I.

Fig. 7 shows the photograph and schematic drawing of the experimental setup. As shown in Fig. 7, the harvesting unit is installed in a host-structure and excited by a spring. One side of the host-structure is fixed on the ground, and another side is connected to the shake table which 

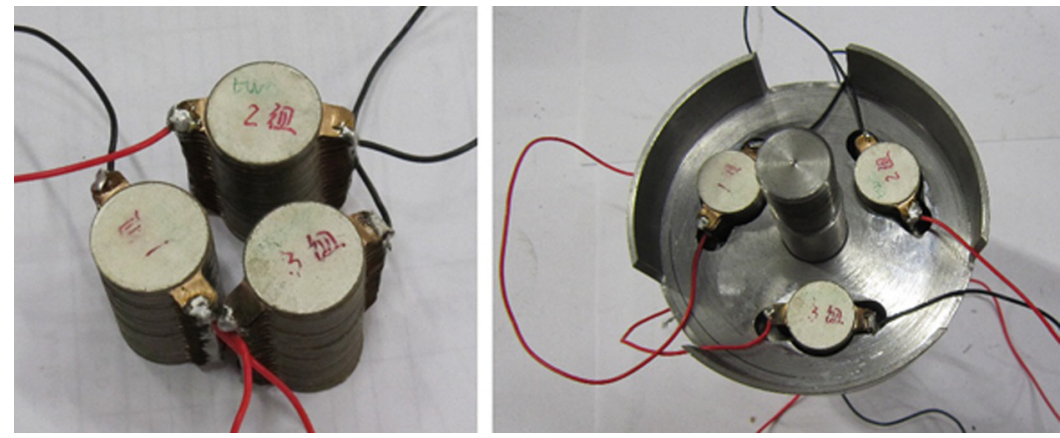

FIG. 6. Piezoelectric stacks and harvesting unit.

TABLE I. Characteristic properties of the piezoelectric stack.

\begin{tabular}{lccc}
\hline \hline Material properties & Value & Stack properties & Value \\
\hline Material type & PZT-8 & Diameter $(\mathrm{mm})$ & 20 \\
Coupling factors $k_{33}$ & 0.68 & Height $h(\mathrm{~mm})$ & 34 \\
Piezoelectric constant $d_{33}(\mathrm{pC} / \mathrm{N})$ & 280 & Single wafer thickness $t(\mathrm{~mm})$ & 0.85 \\
Dielectric constants $\varepsilon_{33} / \varepsilon_{0}$ & 1000 & Layer number & 36 \\
Young's modulus $\mathrm{YE}_{33}\left(10^{10} \mathrm{~N} / \mathrm{m}\right)$ & 7.1 & Mass $m_{\mathrm{p}}(\mathrm{g})$ & 87 \\
Curie point $\left({ }^{\circ} \mathrm{C}\right)$ & 320 & Capacitance $C_{\mathrm{p}}(\mu \mathrm{F})$ & 0.12 \\
Density $\left(10^{3} \mathrm{~kg} / \mathrm{m}^{3}\right)$ & 7.7 & Natural frequency $\omega_{\mathrm{n}}\left(10^{9} \mathrm{~N} / \mathrm{m}\right)$ & 1.1 \\
\hline \hline
\end{tabular}

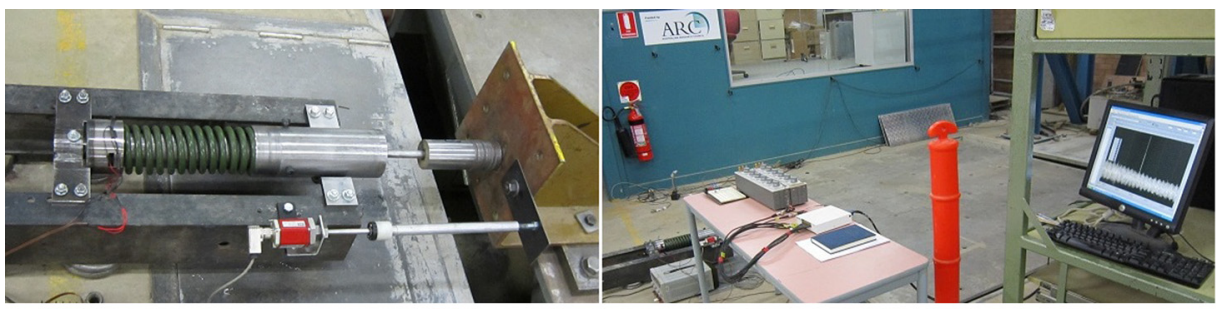

(a)

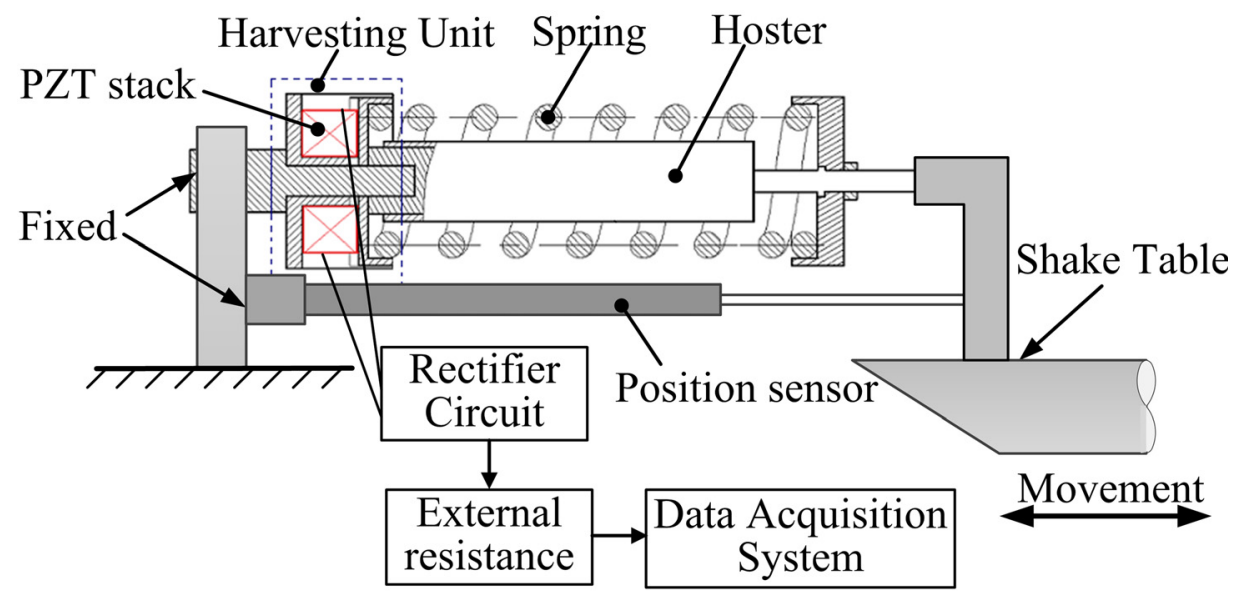

(b)

FIG. 7. Experimental setup (a) photograph and (b) schematic drawing. 


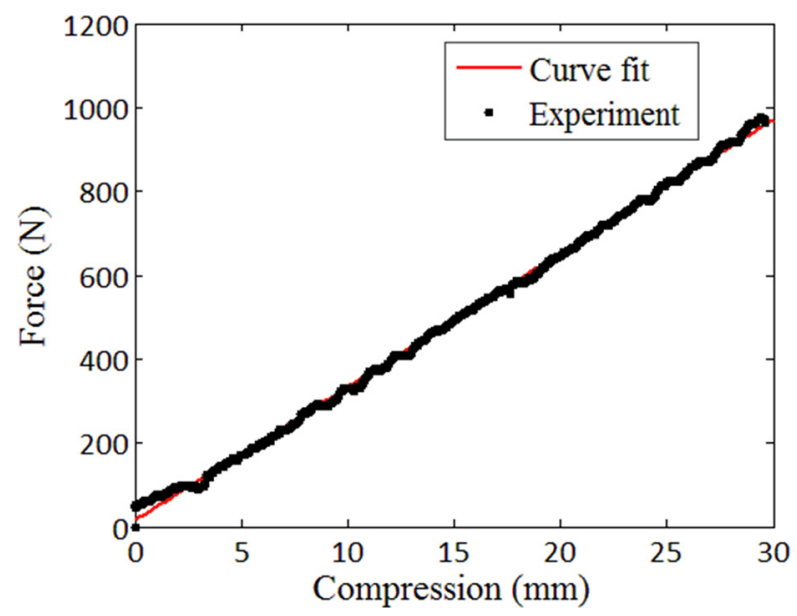

FIG. 8. Spring stiffness static test from a material testing machine.

is used to input the vibration excitations. A spring, as shown in Fig. 7, is used to transfer shake table's motions into vibrational force and apply on the harvesting unit.

As shown in Fig. 2, there are two elastic fixing bolts are used to hold the structure together and applied a pre-compression force to the harvesting units. Therefore, during the test, the shake table first compressed the spring for $50 \mathrm{~mm}$ to apply a pre-loading force on the harvesting unit. Then, the shake table will perform different harmonic motions with different amplitudes (from $10 \mathrm{~m}$ to $40 \mathrm{~mm}$ ), and low frequencies (from $1.0 \mathrm{~Hz}$ to $6.0 \mathrm{~Hz}$ ). Shake table motions will be transferred into large force by the spring due to its large stiffness, i.e., $34 \mathrm{~N} / \mathrm{mm}$, as shown in Fig. 8 . The position sensor is used to measure the movement of shake table in order to calculate the exciting force. The maximum force transmitted by the spring reaches $3060 \mathrm{~N}$ under shake table motion with maximum amplitude of $40 \mathrm{~mm}$. The harvesting unit generates voltages when the excitation from the shake table applies on the wafer-stacks. Rectifier circuit is used to transfer AC voltages generated directly by the harvesting unit into useful DC voltages. Two series-connected rheostats, as shown in Fig. 7, are chosen to serve as the electrical loading of the harvesting unit in order to investigate the relationship between the power output and the external electrical loading. Data acquisition system is used to record the output voltage signal applied on the rheostats.

\section{B. Experimental results comparison and discussion}

Fig. 9 shows the actual exciting force and the real-time output voltage, without the rectifier circuit, of the harvesting unit with $290 \mathrm{k} \Omega$ electrical load when the shake table performs $40 \mathrm{~mm} / 2 \mathrm{~Hz}$ sinusoidal motion. It can be seen that the preloading force is $1700 \mathrm{~N}$ and the

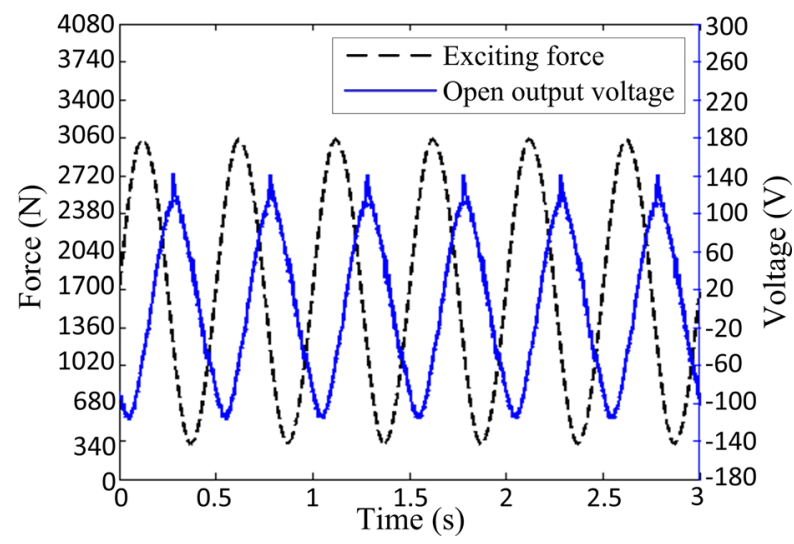

FIG. 9. The applied force vs. output voltage in AC test. 


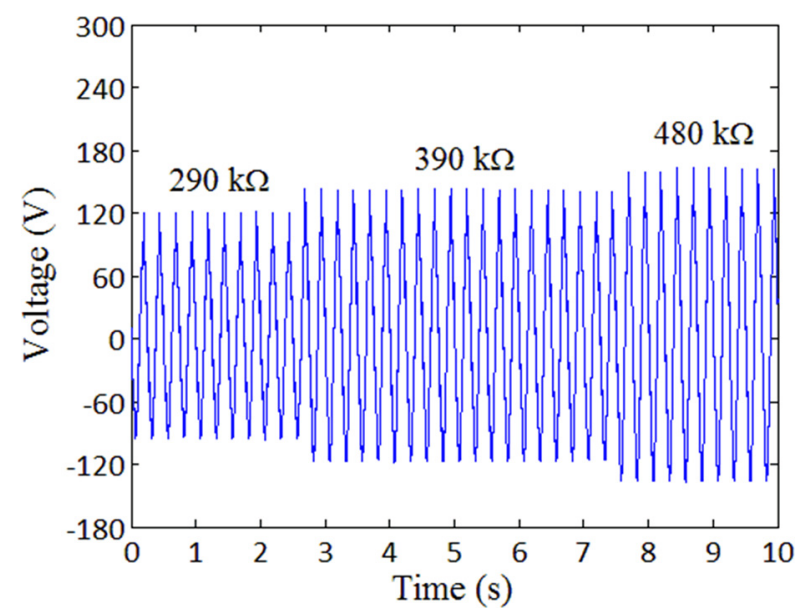

FIG. 10. Output AC voltages.

harmonic force applied on the harvesting unit has $1360 \mathrm{~N}$ amplitude and $2 \mathrm{~Hz}$ frequency when the shake table performs $40 \mathrm{~mm} / 2 \mathrm{~Hz}$ sinusoidal motion. Under this harmonic force, the voltage generated by the harvesting unit is an AC voltage with the same frequency of the excitation. Also, there is a phase change between the excitation and the output AC voltage, which is caused by the response time of the outer-spring and harvesting unit itself.

Under the same input excitation, Fig. 10 shows the output AC voltages under different electrical loads. It can be seen that the amplitude of the output AC voltage increases with the external resistance. Fig. 11 gives the output voltage through the rectifier circuit under different electrical loads. It can be seen that the output voltage through the rectifier circuit is a DC voltage and the value of the DC voltage increases with the external resistance. Figs. 10 and 11 indicate that the output voltage under vibrational excitation relates to external electrical loads.

Fig. 12 gives the relationship between the output DC voltage/power with different external resistive loads under the applied force shown in Fig. 9, i.e., $1360 \mathrm{~N} / 2 \mathrm{~Hz}$. It can be seen that: (1) the output voltage increases with the value of the external resistance and trends to constant after the electrical load exceeding a certain value; (2) the output power firstly increases with the resistance, and then trends to decrease after the external resistance exceeding a certain value. It is obvious that there is an optimal resistance, with which output power reaches maximum. Comparison between the experimental results and the theoretical analysis proves the effectiveness of the electromechanical model.

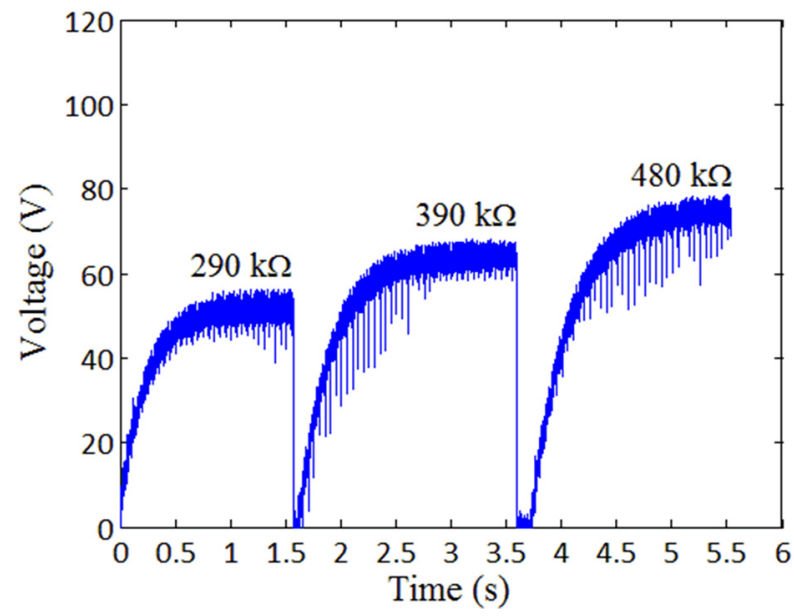

FIG. 11. Output DC voltages. 

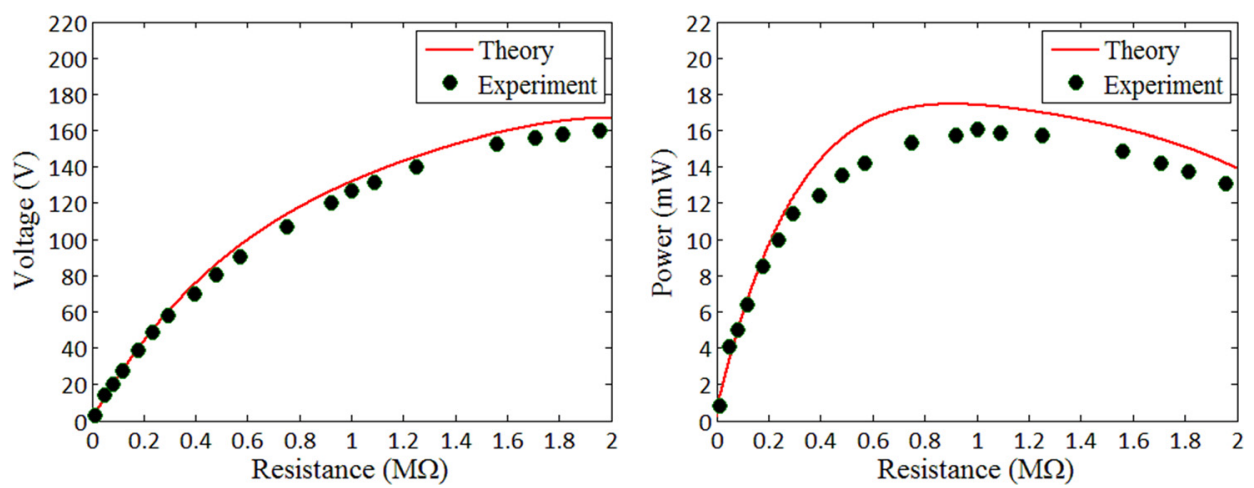

FIG. 12. Output DC voltage/power vs. electrical loads under 1360 N/2 Hz excitation.

Fig. 13 shows the relationship between the output power and the external loads under the excitations with different force amplitudes, i.e., $680 \mathrm{~N}, 1020 \mathrm{~N}$, and $1360 \mathrm{~N}$, and fixed frequency, $2 \mathrm{~Hz}$. It can be obtained that: (1) the output power increases with the amplitudes of exciting forces, and the maximum output power increases, from $3 \mathrm{~mW}$ to $16 \mathrm{~mW}$, when the exciting force amplitude increases from $680 \mathrm{~N}$ to $1360 \mathrm{~N}$; (2) the optimal electrical load does not change its value with the exciting force amplitude under the fixed frequency.

Fig. 14 shows the relationship between the output power and the external loads under the excitations with different frequencies, i.e., $2 \mathrm{~Hz}, 4 \mathrm{~Hz}$, and $6 \mathrm{~Hz}$, and fixed force amplitude, $1360 \mathrm{~N}$. It can be obtained that: (1) the output power increases with the frequency of excitations, and the maximum harvested power increases from $16 \mathrm{~mW}$ to $85 \mathrm{~mW}$, when the exciting frequency increases from $2 \mathrm{~Hz}$ to $6 \mathrm{~Hz}$; (2) the value of the optimal electrical load is inversely proportional to the input frequency of the excitation, and its value decreases from $1050 \mathrm{k} \Omega$ to $350 \mathrm{k} \Omega$, when the exciting frequency increases from $2 \mathrm{~Hz}$ to $6 \mathrm{~Hz}$.

To clearly demonstrate the relationship between the harvested electrical energy with the external excitation, Fig. 15 plots the maximum output power against the input frequency when the exciting force amplitude fixed on $1360 \mathrm{~N}$, and Fig. 16 plots the maximum output power against the exciting force amplitude when the frequency of the excitation fixed on $6 \mathrm{~Hz}$. Based on Figs. 15 and 16, it can be concluded that the electrical energy generated by the harvesting unit is proportional to the frequency of external harmonic excitation and is proportional to the square of the force amplitude of the harmonic excitation. It is reasonable to say that the proposed piezoelectric energy harvester is a linear system, the output power increases with the external excitation level in the considered frequency and force.

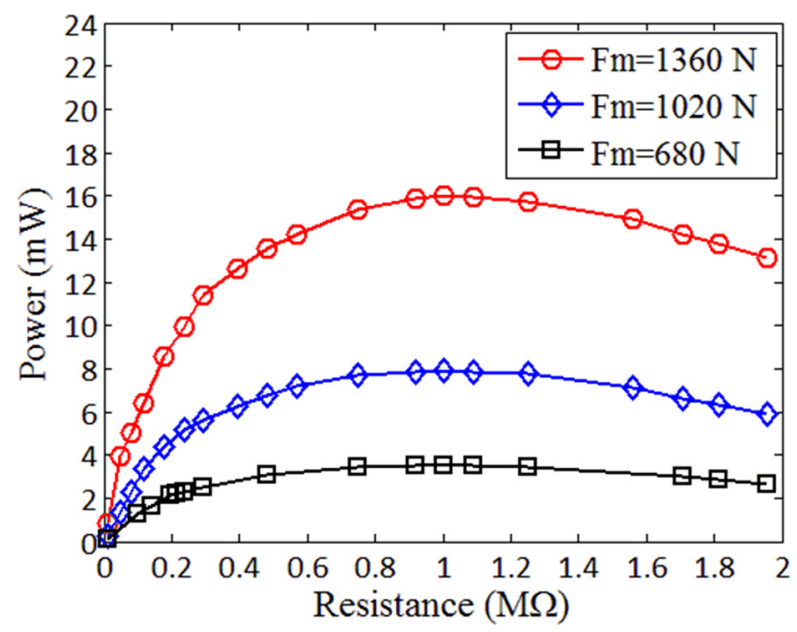

FIG. 13. Output power comparison under same frequency. 


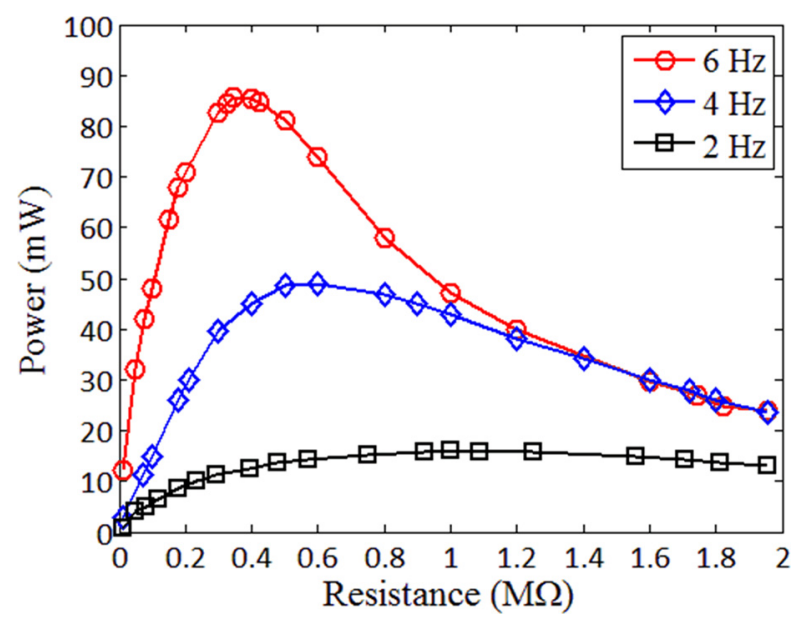

FIG. 14. Output power comparison under same force amplitude.

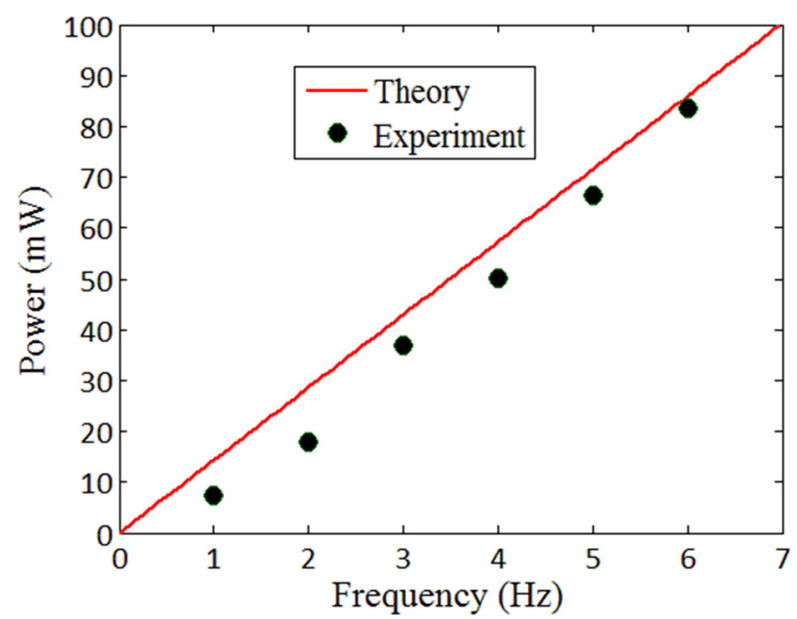

FIG. 15. Maximum output power vs. exciting frequency.

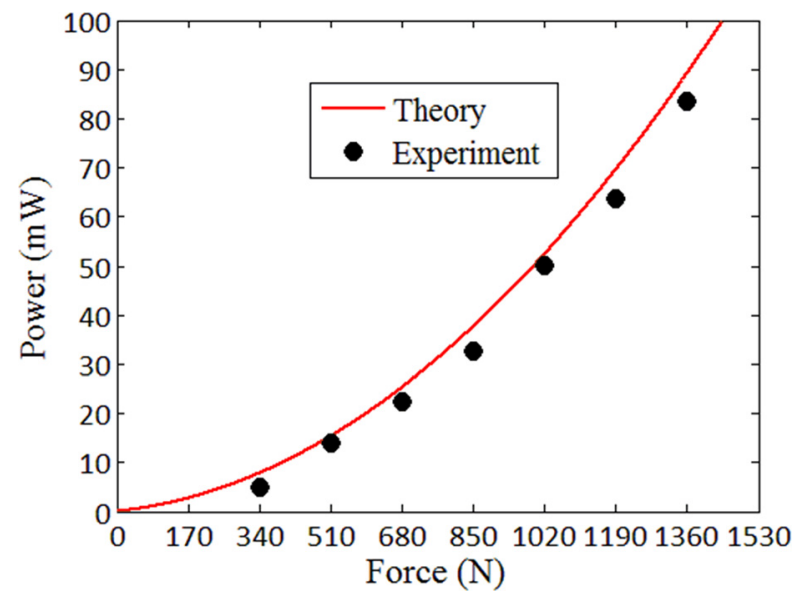

FIG. 16. Maximum output power vs. exciting force amplitude. 


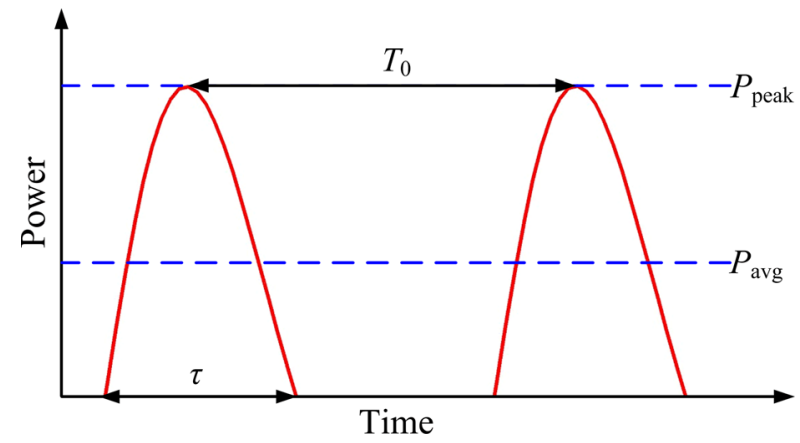

FIG. 17. The pulse of power.

\section{EVALUATION OF THE CAPABILITY OF THE ROADWAY HARVESTER}

Built on the experimental results of the output power of an individual harvesting unit, this section aims to estimate the capability of the designed roadway harvester installed in the road, as shown in Fig. 2. In practice, the electrical power generated by the roadway harvester embedded in the road is a pulse of power, which is registered with each compression cycle (such as the passage of a vehicle tire over the harvester). An example of the power pulse induced by passing a vehicle with two axles over the roadway harvester is shown in Fig. 17. The period $T_{0}$ is determined by the distance between vehicle axles and the vehicle speed, and the pulse length $\tau$ is determined by the tire contact patch length and the vehicle speed. In general, the pulse length $\tau$ is very small $(5 \mathrm{~ms}-100 \mathrm{~ms})$, therefore, the average power $P_{\text {avg }}$ can be calculated approximately as

$$
P_{\text {avg }}=\frac{\tau}{T_{0}} P_{\text {peak }}
$$

According to the Transportation Energy Data Book, ${ }^{18}$ the majority of vehicles on the roadway are cars (two-axle) and light duty trucks (two-axle and four-tire), and cars account for $52.8 \%$ and two-axle trucks account for $37.1 \%$ of the total vehicle-miles traveled in 2011. Also, based on the data about the gross weight of vehicles in Table 5.13 of the Transportation Energy Data Book, the average weight of a single axle of cars and two-axle trucks can be calculated approximately as $10350 \mathrm{~N}$ and $33750 \mathrm{~N}$, respectively. Zhao et al. ${ }^{13}$ indicated that the average contact patch length is $20 \mathrm{~cm}$, and the average distance between the axles of two-axle vehicles is $4.5 \mathrm{~m}$.

Experimental results indicate that the proposed piezoelectric harvesting unit can generate 85 $\mathrm{mW}$ DC power under compressing force with $1360 \mathrm{~N}$ amplitude and $6 \mathrm{~Hz}$ frequency, and the generated power is proportional to the frequency and the square of the amplitude of the compressing force. For a highway, assume that the traffic volume is 2000 vehicle/h, and the average speed of those vehicles is $100 \mathrm{~km} / \mathrm{h}$. Then, the induced pulse of power in the proposed roadway harvester has narrow band ( $\tau$ is about $7.1 \mathrm{~ms}$ ) and the pulse of power occurs every $0.16 \mathrm{~s}$. A power pulse every $0.16 \mathrm{~s}$ would correspond to a compression frequency of $6.2 \mathrm{~Hz}$. Combined with the weight data of the vehicles from the Transportation Energy Data Book, the proposed roadway harvester (shown in Fig. 2) installed in this highway has the ability to generate two pulse powers with $P_{\text {peak }}$ equal to $22.5 \mathrm{~W}$ when passing a two-axle vehicle in $100 \mathrm{~km} / \mathrm{h}$ velocity. Then, based on Eq. (23), the roadway harvester can generate about $1 \mathrm{~W}$ DC power when passing a two-axle vehicle in $100 \mathrm{~km} / \mathrm{h}$ velocity. For the highway with the traffic volume 2000 vehicle/h, it can be estimated that the proposed roadway harvester has the potential energy harvesting capability of $2000 \mathrm{~W} / \mathrm{h}$.

\section{CONCLUSIONS}

Harvesting energy from traffic-induced vibrations to power the remote equipments used in transportation infrastructures is investigated in this paper. A novel roadway energy harvester based on piezoelectric stacks is developed to scavenge energy from traffic-induced vibrations. 
Theoretical analysis verified with experimental results of the output power of the harvesting unit indicates that: (1) the output power depends not only on the harvesting unit itself but also on the external resistive loads. There is an optimal resistance, with which the output power reaches maximum value; (2) the value of the optimal resistances is not affected by the excitation amplitudes, and it is inversely proportional to the exciting frequencies, which agrees with previous theoretical analysis; and (3) the output power increases significantly with the amplitude and frequency of the input excitation, and the maximum harvested power is proportional to the frequency and the square of the amplitude of the external vibration.

Experimental results indicate that one single harvesting unit can generate $85 \mathrm{~mW}$ DC power under the excitation with $1360 \mathrm{~N}$ force amplitude and $6 \mathrm{~Hz}$ frequency. Based on the experimental results, the proposed roadway harvester embedded on the surface of the road has the ability to generate $1 \mathrm{~W}$ DC power when passing a two-axle vehicle in $100 \mathrm{~km} / \mathrm{h}$, and the output power increases with the velocity of the passing vehicle because higher velocity means higher exciting frequency. It clearly indicates that the electric power scavenged power vehicle is enough for powering common lower-power-needed remote equipments such as wireless sensors system. In addition, for a certain highway with 2000 vehicle/h traffic volume, the proposed roadway harvester has the potential energy harvesting capability of $2000 \mathrm{~W} / \mathrm{h}$. This huge harvested electrical power may also be used to power normal electrical equipment on the roadway, such as lighting system, emergency communication unit, and roadside advertisement.

\section{ACKNOWLEDGMENTS}

This work was supported by the National Science Foundation of China under Grant No. 51175265 .

${ }^{1}$ N. G. Elvin, N. Lajnef, and A. A. Elvin, "Feasibility of structural monitoring with vibration power sensors," Smart Mater. Struct. 15, 977-986 (2006).

${ }^{2}$ G. Park, T. Rosing, M. D. Todd, C. R. Farrar, and W. Hodgkiss, "Energy harvesting for structural health monitoring sensor networks," J. Infrastruct. Syst. 14, 64-67 (2008).

${ }^{3}$ D. B. Ashebo, C. A. Tan, J. Wang, and G. Li, "Feasibility of energy harvesting for powering wireless sensors in transportation infrastructure applications," Proc. SPIE 6934, 69340Y1-8 (2008).

${ }^{4}$ C. B. Williams and R. B. Yates, "Analysis of a micro-electric generator for microsystems," Sens. Actuators, A 52, 8-11 (1996).

${ }^{5}$ X. Z. Jiang, J. Wang, Y. C. Li, and J. C. Li, "Design and modeling of a novel linear electromagnetic vibration energy harvester," Int. J. Appl. Electromagn. Mech. (published online 2014).

${ }^{6}$ S. Boisseau, G. Despesse, and A. Sylvestre, "Optimization of an electrets-based energy harvester," Smart Mater. Struct. 19, 075015 (2010).

${ }^{7}$ D. A. Wang and N. Z. Liu, "A shear mode piezoelectric energy harvester based on a pressurized water flow," Sens. Actuators, A 167, 449-458 (2011).

${ }^{8}$ S. R. Anton and H. A. Sodano, "A review of power harvesting using piezoelectric materials (2003-2006)," Smart Mater. Struct. 16, R1-R21 (2007).

${ }^{9}$ X. Z. Jiang, Y. C. Li, and J. C. Li, "A piezoelectric-wafer-stack vibration energy harvester for wireless sensor networks," Proc. SPIE 8692, 869246 (2013).

${ }^{10}$ M. Peigney and D. Siegert, "Piezoelectric energy harvesting from traffic-induced bridge vibrations," Smart Mater. Struct. 22, 095019 (2013).

${ }^{11}$ M. Wischke, M. Masur, M. Kroner, and P. Woias, "Vibration harvesting in traffic tunnels to power wireless sensor nodes," Smart Mater. Struct. 20, 085014 (2011).

${ }^{12}$ H. C. Xiong, L. B. Wang, D. Wang, and C. Druta, "Piezoelectric energy harvesting from traffic induced deformation of pavements," Int. J. Pavement Res. Technol. 5(5), 333-337 (2012).

${ }^{13} \mathrm{H}$. Zhao, J. Ling, and J. Yu, "A comparative analysis of piezoelectric transducers for harvesting energy from asphalt pavement," J. Ceram. Soc. Jpn. 120, 317-323 (2012).

${ }^{14} \mathrm{H}$. Zhao, J. Yu, and J. Ling, "Finite element analysis cymbal piezoelectric transducers for harvesting energy from asphalt pavement," J. Ceram. Soc. Jpn. 118, 909-915 (2010).

${ }^{15}$ H. J. Xiang, J. J. Wang, Z. F. Shi, and Z. W. Zhang, "Theoretical analysis of piezoelectric energy harvesting from traffic induced deformation of pavements," Smart Mater. Struct. 22, 095024 (2013).

${ }^{16}$ IRE "Standards on piezoelectric crystals," in Proceedings of the IRE (1949), Vol. 37, pp. 1378-1395.

${ }^{17}$ J. G. Smits, "Iterative method for accurate determination of the real and imaginary parts of the materials coefficients of piezoelectric ceramics," IEEE Trans. Sonics Ultrason. 23, 393-402 (1976).

${ }^{18}$ S. C. Davis, S. W. Diegel, and R. G. Boundy, Transportation Energy Data Book, 32 ed. (Oak Ridge National Labs ORNL, 2013), Vol. 6989. 
Journal of Renewable \& Sustainable Energy is copyrighted by the American Institute of Physics (AIP). Redistribution of journal material is subject to the AIP online journal license and/or AIP copyright. For more information, see

http://jrse.aip.org/about/rights_and_permissions. 